\title{
Clinical Profile and Outcome of Necrotizing Fasciitis
}

\author{
Pathak $A^{1}$, Khadka DB ${ }^{2}$, Gautam $S^{3}$, Sharma $A^{4}$, Bahl DV
}

\begin{abstract}
Background: Necrotizing fasciitis is soft tissue infection which spreads through the fascial planes. It is a surgical emergency with its rapid onset and varied occult characteristic can cause diagnostic delays resulting in high mortality and morbidity. Objectives: To study the clinical profile and evaluate the determinants of outcome in patients with necrotizing fasciitis. Methods: A descriptive study of patients admitted and operated in the surgical units of Nepalgunj Medical College and Teaching Hospital Kohalpur with a diagnosis of necrotizing fasciitis from January 2013 to December 2015. Results: Total of 45 patients were included in the study. There were $27(60 \%)$ male and $18(40 \%)$ female, male to female ratio being 1.5: 1 . The patient's age ranged from 2 to 76 years with mean age being $48.04 \pm 19.62$ years. $38(84.44 \%)$ patients had no predisposing factor however trauma was the triggering factor in $4(8.89 \%)$ cases followed by surgery and insect bite. Diabetes mellitus 26(57.78\%) and alcohol dependence 5(11.11\%) cases were the most common co morbid condition associated with the disease. The lower limb 28(62.22\%) was the most common site followed by upper limb, scrotum, perineum, trunk and abdomen. The growth was polymicrobial in $24(53.33 \%)$, mono microbial in $16(35.56 \%)$ and sterile in 5(11.11) cases. Staphylococcus aureus was predominant in 24(53.33\%) cases followed by Streptococcus, E coli, Klebsiella, Enterococcus. The main procedure was debridement with secondary suturing $23(51.11 \%)$ and debridement with grafting in $18(40 \%)$ patient. Amputation was done in 2(4.44\%) and secondary wound healing was observed in $2(4.44 \%)$ patients. $39(86.67 \%)$ cases improved with the above management while mortality was observed in $3(6.67 \%) .3(6.67 \%)$ cases left against medical advice so outcome was unknown Conclusion: On the basis of our study we concluded that high index of suspicion and awareness of the clinical presentation is required to diagnose this condition early. Diabetes mellitus is the most common co-morbid condition associated with necrotizing fasciitis. The presence of multiple co morbid conditions and delayed presentation reduces the survival rate. Early diagnosis and debridement, broad spectrum antibiotics with organ supportive intensive care will improve morbidity and mortality.
\end{abstract}

Key words: Diabetes mellitus, debridement, necrotizing fascitis

\section{INTRODUCTION}

Necrotizing fasciitis is fatal life threatening soft tissue infection which is progressive and spreads rapidly through the fascial planes. It is a surgical emergency with increased mortalty rate. The varied clinical presentation includes pain out of proportion to the physical finding, haemorrhagic bullae, edema, subcutaneous bleeding, ulcer and gangrene. Presence of systemic manifestation like hypotension, fever, tachycardia, tachypnea and mental disturbances further adds to the morbidity and mortality. Mortality rate has not changed significantly for several decades, and still remains high, ranging from $24-34 \%{ }^{1}$.
1. Dr. Anand Pathak
2. Dr. D. B. Khadka
3. Dr. Subodh Gautam
4. Dr Anup Sharma
5. Prof. Dig Vijay Bahl

Address for correspondence:

Dr. Anand Pathak

Department of Surgery

Nepalgunj Medical College Teaching Hospital,

Kohalpur, Banke, Nepal

Email: ananda_pathak@yahoo.com
The term necrotizing fasciitis was coined by Wilson ${ }^{2}$ in 1952 and referred it to as any infection of the soft tissue that is associated with necrosis requiring operative intervention. The purpose of the present study was to evaluate the common clinical presentation and the factors determining the mortality and morbidity of this disease in this part of Nepal.

\section{MATERIALS AND METHOD}

A descriptive study was conducted at the surgical units of Nepalgunj Medical College and teaching hospital Kohalpur from January 2013 to December 2015. All patients presenting with necrotizing fasciitis were included in the study. Clinical history and detailed examination, co morbidity, predisposing factor, duration of symptoms, site of involvement was noted. Biochemical parameters like white blood cell count, hemoglobin, renal parameters, serum levels of sodium, albumin and sugar at the time of admission was recorded. The presence of grayish necrotic fascia, demonstration of a lack of resistance of normally adherent muscular fascia to blunt dissection, lack of bleeding of the fascia during dissection, the presence of foul-smelling "dish-water" pus with obliterative thrombosis of arteries and veins were used for the diagnosis of the disease.

Patients were managed initially with broad spectrum antibiotics followed by culture-based antimicrobials along with 
debridement and repeated as required till the devitalized tissues were completely removed. Daily dressings followed by either secondary suturing or grafting of the wound were done. The time from admission to operative treatment, type of operative intervention, the culture and sensitivity reports, the need for amputation, the duration of hospitalization and mortality rate were recorded

\section{RESULTS}

Total of 45 patients were included in the study. There were $27(60 \%)$ male and $18(40 \%)$ female, male to female ratio being 1.5: 1 . The patient's age ranged from 2 to 76 years with mean age being $48.04 \pm 19.62$ years. The highest number of patients, $13(28.89 \%)$ were in the age group of 51 to 60 years as shown in table I.

\begin{tabular}{|c|c|c|}
\hline Age in years & Number $(\mathbf{n}=\mathbf{4 5})$ & Percentage \\
\hline $1-10$ & 3 & 6.67 \\
\hline $11-20$ & 3 & 6.67 \\
\hline $21-30$ & 3 & 6.67 \\
\hline $31-40$ & 2 & 4.44 \\
\hline $41-50$ & 8 & 17.78 \\
\hline $51-60$ & 13 & 28.89 \\
\hline $61-70$ & 11 & 24.44 \\
\hline $71-80$ & 2 & 4.44 \\
\hline
\end{tabular}

Table I: Age distribution

Out of 45 patients, $38(84.44 \%)$ had no predisposing factor. Among the predisposing factor trauma was the most common cause in $4(8.89 \%)$. Other factors being surgery and insect bite as shown in table II.

\begin{tabular}{|c|c|c|}
\hline $\begin{array}{c}\text { Predisposing } \\
\text { factor }\end{array}$ & Number(n=45) & Percentage \\
\hline Idiopathic & 38 & 84.44 \\
\hline Trauma & 4 & 8.89 \\
\hline Surgery & 2 & 4.44 \\
\hline Insect bite & 1 & 2.22 \\
\hline
\end{tabular}

Table II: Predisposing factor with frequency

The associated co morbidities as shown in table III revealed that diabetes mellitus was the most common which was found to be in $26(57.78 \%)$ patients followed by alcohol dependance in $5(11.11 \%)$ cases. However no co morbidities were found in $7(15.56 \%)$ cases. The lower limb $28(62.22 \%)$ was the most common site followed by upper limb, scrotum, perineum, trunk and abdomen. All patients had pain, tenderness, erythema and warm skin. 19(42.22\%) also had bulla formation.

\begin{tabular}{|c|c|c|}
\hline Co morbidities & Number( $\mathbf{n = 4 5})$ & Percentage \\
\hline Diabetes mellitus & 26 & 57.78 \\
\hline Alcohol addiction & 5 & 11.11 \\
\hline Anemia & 2 & 4.44 \\
\hline Immunosuppressant & 2 & 4.44 \\
\hline IV drug abuse & 1 & 2.22 \\
\hline Liver disease & 1 & 2.22 \\
\hline Renal disease & 1 & 2.22 \\
\hline No comorbidities & 7 & 15.56 \\
\hline
\end{tabular}

Table III: Associated co morbidities with frequency

The bacteriological study revealed that growth was polymicrobial in $24(53.33 \%$ ) and mono microbial in $16(35.56 \%)$ patients, however in $5(11.11 \%)$ cases it was sterile. The common growth of organism found was Staphylococuus aureus in $24(53.33 \%)$ cases followed by Streptococcus, E coli, klebsiella, Enterococcus. Pseudomonas was isolated in a single case. Staphylococcus aureus was the most predominant organism found in polymicrobial growth in $18(40 \%)$ cases whereas Group A Beta hemolytic streptococci was predominant organism in monomicrobial growth in $12(26.67 \%)$ cases.

Out of 45 patients, $23(51.11 \%)$ patients underwent debridement with secondary suturing and debridement with grafting was done in $18(40 \%)$ patients. Amputation was done in $2(4.44 \%)$ patients to control the rapidly progressing infection. Secondary wound healing was observed in $2(4.44 \%)$ patients.

The aggressive and early intervention led to the improvement in $39(86.67 \%)$ cases. Mortality was observed in $3(6.67 \%)$ cases out of which two had uncontrolled diabetes mellitus and one patient had renal disease. The cause of death was sepsis and multi organ failure in these cases. Outcome in $3(6.67 \%)$ patients were unknown as they left against medical advices.

\section{DISCUSSION}

Necrotizing fasciitis is progressive fulminant bacterial infection of subcutaneous fat which spreads rapidly through the fascial plane causing extensive tissue damage. It is a surgical emergency and has a cumulative rate of mortality of $34 \%(6-$ $76 \%)^{3}$. Similar finding of mortality of $21.9 \%$ was noted by Anogoue et $\mathrm{al}^{4}$ in his systemic review of necrotizing fascitis. The difficulty of making early diagnosis and lack of cutaneous manifestation delays the surgical intervention leading to increased morbidity and mortality.

The majority of patients presented with pain which was out of proportion to apparent physical finding. Tenderness, erythematic and warm skin was present in all cases which is similar to the clinical profile described by Hasham et al ${ }^{5}$ and Hassell et $a^{6}$ in their studies. Clinically pain precedes skin changes by $24-48$ hours and apparently normal looking skin is 
seen during the early pathological stages despite extensive infection underlying the fascia. An intermediate stage characterized by the formation of small bullae was noted as the disease progressed. Large haemorrhagic bullae, skin ulceration crepitation and motor and sensory deficits were late signs; often called as the "hard sign" of disease?.

Patients with necrotizing fasciitis may have some initiating factor such as trauma, surgery, scratch or bites ${ }^{8}$. However in our study $84.44 \%(38)$ patients had no predisposing cause while in remaining cases trauma $8.89 \%(4)$, surgery $4.44 \%(2)$ and insect bite in $2.22 \%(1)$ was found as an initiating factor which is in accordance with study done by Garg et $\mathrm{al}^{9}$.

Wong et al. ${ }^{10}$ in 2003 found that diabetes mellitus(71\%) was the most common co morbidity followed by alcoholism(10.4\%) which was similar to our study in which $26(57.78 \%)$ and $5(11.11 \%)$ had diabetes mellitus and alcoholism respectively. Out of 45 cases; $7(15.56 \%)$ had no associated disease. Anemia, chronic renal disease and use of immunosuppressant were other factor.

In our study, the lower limb was most commonly affected site in $62.22 \%$ (28) cases followed by upper limb $13.33 \%$ (6), scrotum $8.89 \%(4)$ perineum $6.67 \%$ (3) trunk $6.67 \%$ (3) and abdomen $2.22 \%(1)$. Similar findings were noted by Frazee et al $^{11}$ in 2008 and Nisbet et a $^{12}$ in 2012 where the involvement of extremities was seen in $73.7 \%$ and $70 \%$ respectively

The pattern of growth in our study was of polymicrobial $53.33 \%$ (24) followed by monomicrobial in $35.56 \%$ (16). $11.11 \%$ (5) of cases did not show growth. The common organisms found were Staphylococuus aureus in $53.33 \%$ (24) cases followed by Streptococcus, E coli, Klebsiella, Enterococcus. Pseudomonas was isolated only in a single case. Staphylococcus aureus was the most predominant organism found in polymicrobial growth in $40 \%$ (18) cases whereas Group A Beta hemolytic Streptococci was common in monomicrobial growth in $26.67 \%$ (12) cases. Similar profile of growth of organism was found by Elliot et al. ${ }^{13}$ and Brook et al. ${ }^{14}$ in their studies respectively.

In present study $51.11 \%$ (23) patient underwent debridement with secondary suturing and debridement with grafting in $40 \%$ (18) patient. Amputation was done in $4.44 \%(2)$ patient to control the rapidly progressing infection. Secondary healing of wound was observed in $4.44 \%(2)$ patients. Similar was the outcome in the study conducted by Madhumita et al. ${ }^{15}$ in 2011 where $48 \%$ of the patients underwent debridement with secondary suturing while $42 \%$ of the patients required skin grafting.

Our preference for the aggressive and early intervention led to the improvement in $86.67 \%$ (39) cases. Mortality was observed in $6.67 \%$ (3) cases out of which two had uncontrolled diabetes mellitus and one patient had renal disease. The cause of death was sepsis and multi organ failure in those cases. Outcome in
6.67\%(3) patients were unknown as they left against medical advices. The mortality rate in studies conducted by Wong et al. ${ }^{10}$ and Elliot et al. ${ }^{13}$ was similar to our study.

\section{CONCLUSION}

Necrotizing infection remains one of the most devastating soft tissue polymicrobial infections in surgical practice. It is more common in middle aged group with diabetes mellitus being the most common morbidity associated with it. A high index of suspicion should be made if there is rapidly progressing swollen tender erythematous lesion with bulla and crepitation. Early surgical debridement under broad antibiotic coverage with dressing is the cornerstone of therapy. The presence of multiple comorbidity and delay in the diagnosis and intervention increases the morbidity and mortality.

\section{REFERENCES}

1. Yaghubiian A, de Virgillio C, Dauphine C, Lewis R, Lin M. Use of Admission Serum Lactate and Sodium Levels to Predict Mortality in Necrotizing Soft-Tissue Infections. Arch Surg 2007; 142(9):8406.

2. Wilson B. Necrotizing fasciitis. Am Surg 1952; 18(4):416-31.

3. McHenry CR, Piotrowski JJ, Petrinic D, Malangoni MA. Determinants of mortality for necrotizing soft-tissue infections. Ann Surg. 1995; 221:558-65.

4. Angoules AG, Kontakis G, Drakoulakis E, Vrentzos G, Granick MS, Giannoudis PV. Necrotizing fasciitis of upper and lower limb: a systematic review. Injury 2007; 38S:18-25.

5. Hasham S, Matteucci, P, Stanley P. Necrotizing fasciitis. BMJ 2005; 330:830-3.

6. Hassell M, Fagan P, Carson P. Streptococcal necrotizing fasciitis from diverse strains of Streptococcus pyogenes in tropical northern Australia: case series and comparison with the literature. BMCInfect Dis 2004; 4:60.

7. Weiss KA, Laverdiere M. Group a streptococcus invasive infections: a review. Can J Surg. 1997; 40(1):18-25.

8. Singh G, Sinha SK, Adhikary S, Babu KS, Ray P, Khanna SK. Necrotizing infection of the soft tissueu: A clinical profiles. Eur J Surg 2002;168:366-71.

9. Garg CP, Patel RN, Patel DN, Anajwala PH. Necrotizing Fasciitis: A prospective clinical study. Gujrat Med J 2009; 64(2): 55-8.

10. Wong $\mathrm{CH}$, Chang HC, Pausypathy S, Khin LW, Tan JL, Low CO. Necrotizing fasciitis: clinical presentation, microbiology, and determinants of mortality. J Bone Joint Surg Am 2003; 85(8):1454-60.

11. Frazee BW, Fee C, Lynn J, Wang R, Bostrom A, Hargis C. Community-acquired necrotizing soft tissue infections: a review of 122 cases presenting to a single emergency department over 12 years. J Emerg Med 2008; 34(2):139-46.

12. Nisbet M, Ansell G, Lang S, Taylor S, Dzendrowskyj P, Holland D. Necrotizing fasciitis: review of 82 cases in South Auckland. Intern Med J 2011;41(7):543-8.

13. Elliott $D$, Kufera JA, Myers RA. The microbiology of necrotizing soft tissue infections. Am J Surg 2000;179(5):361-6.

14. Brook I, Frazier EH. Clinical and Microbiological Features of Necrotizing Fasciitis. J Clin Microbiol 1995; 33(9):2382-7.

15. Madhumita M, Anil KS, Ranu RB, Swapan B. A Clinicopathological study of necrotizing fasciitis. Al Ameen J Med Sci 2011; 4(1):6-13. 